\title{
Análisis de fiabilidad y estructura factorial de la versión española del TPQ - Cuestionario de Percepción del Tratamiento en Drogodependencias
}

\author{
Ioseba Iraurg( ${ }^{(1)}$; Merte Rodríguez ${ }^{(2,7)}$; Irene Carreras Prieto ${ }^{(2,7)}$; Miguel Landabaso ${ }^{(3,7) ;}$ \\ Agustín Larrazabal ${ }^{(4,7)}$; Jesús Muñoz ${ }^{(5)}$; MertXe Estébanez ${ }^{(6)}$ y Juan Manuel Jiménez-Lerma ${ }^{(2,7)}$ \\ (1) Módulo de Asistencia Psicosocial de Rekalde. Bilbo-Bilbao. \\ (2) Centro de Tratamiento de Toxicomanías de Alava. Vitoria-Gasteiz. Osakidetza/Servicio Vasco de Salud. \\ (3) Centro de Drogodependencias de Barakaldo. Bizkaia. Osakidetza/Servicio Vasco de Salud. \\ (4) Centro de Salud Mental de Rentería. Gipuzkoa. Osakidetza/Servicio Vasco de Salud. \\ (5) Director Gerente del Hospital Psiquiátrico y de la Red de Salud Mental Extrahospitalaria de Alava. Osakidetza/Servicio Vasco de Salud. \\ (6) Directora Médica del Hospital Psiquiátrico y de la Red de Salud Mental Extrahospitalaria de Alava. Osakidetza/Servicio Vasco de Salud. \\ (7) Asociación Vasca de Drogodependencias / Drogamenpekotasunerako Euskal Elkartea (AVD-DEE)
}

\section{RESUMEN}

El "Treatment Perceptions Questionnaire (TPQ)" es una escala breve y autoadministrada cuyo propósito es la valoración de la satisfacción de usuarios de drogas respecto al programa de tratamiento en el que son asistidos. El objetivo del estudio es analizar la consistencia interna y la fiabilidad de la versión española de esta escala, así como su validez de constructo. Para ello se ha aplicado el TPQ a 100 usuarios de un Centro de Tratamiento de Drogodependencias. Los resultados, registrados y codificados según los algoritmos originales, fueron sometidos a un análisis factorial de componentes principales resultando tres factores que en conjunto expresaban el $58,6 \%$ de la varianza explicada (VE). El primer factor, denominado "disponibilidad y confianza en el equipo", asumía el 33,4\% de VE y presentaba un alpha de Cronbach $(\alpha)$ de 0,62 y un Coeficiente de Correlación Intraclase (CCI) de 0,80. El segundo factor, con el 13,9\% de $V E$, hace alusión a la "confianza y satisfacción con el equipo y programa", y presenta un $\alpha=0,71$ y un $\mathrm{CCl}=0,85$. Por último, el tercer factor, con un una VE del $11,2 \%$, un $\alpha=0,56$ y un $\mathrm{CCl}$ de 0,81 , agrupa ítems que hacen referencia a la "recepción de información y motivación para el tratamiento." Estos resultados no confirman las dimensiones halladas por los autores del TPQ, planteándose que la satisfacción con el tratamiento ha de ser contemplada y valorada desde un mayor número de áreas implicadas en dicho proceso.

Palabras clave: Fiabilidad, Validez, Satisfacción, Tratamiento, Drogas, TPQ.

\section{SUMMARY}

The Treatment Perception Questionnaire' (TPQ) is a brief, self-administered scale designed to measure drug user satisfaction with the treatment programme they are following. The aim of this work is to analyse the internal consistency and reliability of the Spanish version of the TPQ, as well as the construct validity. A sample group of one hundred heroin users, diagnosed as opium dependents, treated in a Drugs Treatment Centre, was evaluated with TPO. The results, registered and coded according to the original algorithms, were subjected to a principal components factor analysis (PCFA) with varimax rotation. The PCFA revealed a three factors solution stating $58.6 \%$ of the Explained Variance (EV). The first factor, named "readiness and trust in the treatment team", assumed $33.4 \%$ EV and presented a Cronbach coefficient ( $\alpha$ ) of 0.62 and an Intraclass Correlation Coefficient (ICC) of 0.80 . The second factor, with $13.9 \%$ of $E V$, referring to "trust and satisfaction with the treatment team and programme", presents a $\alpha=0.71$ and an ICC $=0.85$. Lastly, the third factor, with an $11.2 \%$ of EV $(\alpha=0.56$ and ICC $=0.81)$ groups together items which relate to "information reception and motivation for the treatment."These results do not confirm the scope found by the TPQ authors, indicating that satisfaction with treatment must be addressed and assessed from a wider number of areas involved in such a process.

Key words: Reliabitity, Validity, Satisfaction, Treatment, Drugs, TPQ. 


\section{INTRODUCCIÓN}

E los últimos años, el estudio de la satisfacción respecto a los servicios sanitarios se ha convertido en un instrumento ampliamente utilizado tanto para la investigación como para el marketing en sistemas de salud. Como bien sugiere Caminal (1), 'el hecho de que los proveedores de estos servicios y algunos investigadores acepten que la satisfacción del paciente es un resultado importante del trabajo realizado por los profesionales, y un factor asociado a la utilización y a la conducta del paciente, justifica que ésta se haya incorporado como una medida de la mejora de la calidad ${ }^{(1-4)}$. No obstante, la calidad en el ámbito de la salud no sólo depende de la actuación médico-sanitaria, sino también está estrechamente relacionada con la infraestructura del servicio y las condiciones de los usuarios, receptores y perceptores del servicio. A este respecto, algunos autores ${ }^{(5)}$ han sugerido que para evaluar la calidad de un servicio, desde el momento mismo de la indicación y planificación del tratamiento, es importante considerar la respuesta y percepción del paciente acerca de la relación terapéutica, de la competencia del equipo de atención, las expectativas ante el tratamiento y el nivel de satisfacción experimentado. Asimismo, otros atributos tales como la correcta prestación de servicios, la presteza de los profesionales para resolver problemas, la atención de los diversos contenidos en la demanda, la cortesía y calidez en la atención, la accesibilidad del servicio, la comodidad de las instalaciones, el tiempo de espera, etcétera, serían todos ellos elementos a contemplar.

En el ámbito de las toxicomanías se han realizado varios esfuerzos por aproximarse a la valoración de la satisfacción por parte de los usuarios ${ }^{(6-8)}$, entre los cuales se ha utilizado como instrumento de evaluación el cuestionario de percepción del tratamiento (TPQ según sus siglas en inglés) ${ }^{(9)}$.

EI TPQ (Treatment Perceptions Questionnaire), desarrollado por el grupo de Marsden en $1998{ }^{(9)}$, es un cuestionario destinado a población en tratamiento por adicción a drogas diseñado para medir la satisfacción del usuario respecto a su proceso terapéutico. El análisis factorial del cuestionario que realizaron sus autores mostró dos dimensiones, cada una compuesta por cinco ítems. El primer factor recoge la percepción de los usuarios sobre la naturaleza y la intensidad del contacto con el personal del programa (Dimensión Equipo Terapéutico) y el segundo factor agrupa los aspectos del servicio de tratamiento, su aplicación, reglas y normativas (Dimensión Programa Terapéutico). Esta bidimensionalidad permite a los autores proponer la obtención de dos puntuaciones diferenciadas atendiendo al sumatorio de los cinco ítems que componen cada dimensión, oscilando las puntuaciones en cada caso entre 0 y 20 puntos. Asimismo, se puede obtener una puntuación total como resultado de la suma de las dos subescalas, o en su caso, por la suma total de los 10 ítems que componen el TPQ, situándose el recorrido de puntuaciones posibles entre 0 y 40 puntos.

Dos grupos han trabajado con la adaptación al castellano, ERIT (6) y Jiménez-Lerma ${ }^{(7)}$, pero hasta el momento no han sido publicadas las características psicométricas de este instrumento, siendo éste el objetivo del presente estudio.

\section{MÉTODO}

Ubicación.- El estudio se ha desarrollado en el Centro de Tratamiento de Toxicomanías de Alava (CTT). Éste es un dispositivo sanitario público dedicado a la atención de usuarios diagnosticados de Trastornos por Abuso de Sustancias no alcohólicas en régimen ambulatorio.

Muestra.- El estudio se centra en la valoración que han realizado 100 usuarios del centro, 50 incluidos en el Programa de Mantenimiento con Metadona (PMM) y otros 50 en el Programa Libre de Drogas (PLD), respecto a la satisfacción que tienen en cuanto a la atención y tratamiento recibido, medida con el 'Treatment Perceptions Questionnaire' (TPQ) ${ }^{(9)}$.

Diseño y procedimiento.- El estudio se ha llevado a cabo mediante un diseño descriptivo de corte transversal en dos fases utilizando métodos de autoinforme (cuestionario) para valorar la satisfacción con el programa.

El único criterio para participar en el estudio fue que los usuarios llevasen en el programa un mínimo de 15 días, para facilitar que los pacientes tuvieran un conocimiento mínimo respecto al funcionamiento de su programa de tratamiento. Dos monitores del centro se ocuparon de solicitar la participación, entregar el cuestionario y trasmitir las pautas de cumplimentación. Todos los individuos fueron informados de que se procedía a conocer cuál era su opinión respecto a la atención y características del centro y que se trataba de un cuestionario anónimo. La entrega de cuestionarios se realizó sistemáticamente hasta conseguir una muestra de 50 cuestionarios por programa; un total de 100 en el centro. La participación en el estudio fue voluntaria, negándose seis usuarios a contestar el cuestionario. En una segunda fase, los mismos sujetos fueron invitados a cumplimentarlo siendo el propósito de esta segunda administración la valoración de la fiabilidad test-retest del instrumento.

Análisis.- Para la descripción de las características de la muestra y de los indicadores de satisfacción se utilizaron la frecuencia (n) y los porcentajes (\%) y/o los estadísticos de tendencia central (media -M- y desviación estándar -DE-) según el tipo de variables consideradas, nominales vs intervalo, respectivamente. 
Con el objeto de estudiar la estructura dimensional del TPQ se realizó un análisis factorial de componentes principales con rotación varimax como estrategia exploratoria para el total de ítems que configuran el TPQ. Este tipo de análisis factorial tiene como objetivo analizar una serie de ítems, variables o fenómenos estudiando cómo están correlacionados entre sí e intentando ver si existe una estructura más simple que la que supone la diversidad de los ítems y/o variables. Se acepta que las intercorrelaciones altas entre un grupo de ítems/variables se deben a ciertos factores o variables generales que representan a dicho grupo ${ }^{(10)}$. Para comprobar el grado de interrelación de las variables se calculó el índice Kaiser-Meyer-Olkin (KMO) y la prueba de esfericidad de Bartlett $\left(\chi^{2}\right)$. Se consideraron aquellos factores/dimensiones con valores propios (eigenvalue) superiores a 1. Para la interpretación de las dimensiones subyacentes se consideraron aquellos ítems con una carga factorial con saturación superior a $0,40\left(R^{2}=0,16\right)$. Desde esta perspectiva, la aplicación de este análisis a la escala TPQ nos permitiría explorar las dimensiones subyacentes y ver si éstas se aproximan a las teóricas.
Para el estudio de la fiabilidad del instrumento se ha analizado la consistencia interna a través del coeficiente alpha $(\alpha)$ de Cronbach. Dicho coeficiente nos informa de hasta qué punto todos los ítems de una escala miden una misma dimensión o, por el contrario, hasta qué punto miden cosas diferentes, pudiéndose corroborar de este modo su significación teórica ${ }^{(10)}$. El coeficiente alfa ha sido calculado tanto para las distintas dimensiones teóricas del TPQ, para la escala total, así como para las posibles dimensiones factoriales encontradas en el estudio.

Para analizar la fiabilidad test-retest se utilizó el coeficiente de correlación intraclase -CCl- (11) utilizando los algoritmos propuestos por Prieto y colaboradores (12-13). Este coeficiente estima la correlación promediada entre todas las posibles ordenaciones de los pares de observaciones y ofrece un índice de concordancia general más ajustado que el coeficiente de correlación de Pearson.

Todos los análisis se llevaron a cabo a través del programa estadístico SPSS ${ }^{(14)}$ en su versión 9.

Tabla 1. - Análisis Factorial del Cuestionario TPQ de 10 ítems ( $n=100)$.

$\begin{array}{lcccc} & \text { Factor } 1 & \text { Factor } 2 & \text { Factor } 3 & \\ \text { Valor Propio (Eigenvalue) } & 3,34 & 1,39 & 1,12 & \text { Factores } \\ \text { Porcentaje de Varianza Explicada } & 33,39 & 13,94 & 11,24 & \text { Teóricos }\end{array}$

1 El equipo no siempre ha comprendido la clase de ayuda que yo quiero.

$0,531 \quad 0,575 \quad$ Equipo

2 He sido bien informado acerca de las decisiones tomadas sobre mi tratamiento.

0,778 Programa

3 El equipo y yo hemos tenido ideas diferentes acerca de cuáles deberían ser los objetivos de mi tratamiento.

0,795

Equipo

4 Siempre ha habido un miembro del equipo disponible cuando yo he querido hablar.

$0,673 \quad 0,414 \quad$ Equipo

5 El equipo me ha ayuda a motivarme para solucionar mis problemas.

$0,423 \quad 0,542 \quad$ Equipo

6 No me han gustado todas las sesiones de tratamiento a las que he asistido.

Programa

7 No he tenido tiempo suficiente para solucionar mis problemas.

8 Yo pienso que los miembros del equipo han sido buenos en sus tareas.

9 He recibido la ayuda que buscaba.

10 No me han gustado algunas de las reglas y normas del tratamiento.

K.M.O. = 0,73; Prueba de Esfericidad de Bartlett: $\chi^{2}=231,30, p<0,001$ 


\section{RESULTADOS}

Las características de la muestra así como el análisis de la satisfacción reflejada por los participantes ha sido recogida en un trabajo previo ${ }^{(7)}$. Se resume a continuación el perfil general del usuario que ha participado en el estudio: prioritariamente hombre $(77 \%)$, con una edad media de 32,9 años ( $\mathrm{DE}=6,5$; recorrido de 18 a 48 años), soltero (57\%), que ha trabajado en mayor o menor medida durante el último año $(54 \%)$, y con un nivel educativo de bachiller elemental o graduado escolar (40\%). En el ámbito de problemas judiciales un $9 \%$ no respondieron a esta cuestión, y de los que lo hicieron, el 62,6\% (57/91) no presentaban antecedentes penales. La heroína, consumida de forma única o en combinación con otra sustancia, fue la sustancia principal que generó la demanda de tratamiento (74\%); con una media de consumo de 11,6 años ( $\mathrm{DE}=6,6$; recorrido 2 a 28 años). Los sujetos vienen recibiendo tratamiento en el CTT durante una media de 27,8 meses ( $D E=32$; recorrido 1 a 156 meses), e iniciaron su primer tratamiento de toxicomanías hace una media de 7,4 años $(D E=6$; recorrido 0 a 26 años). En estas últimas variables el índice de omisiones fue elevado.

\section{Estructura Factorial delTPO}

En la tabla 1 se recogen los datos correspondientes al análisis factorial de componentes principales realizado sobre el TPO. La matriz de correlaciones ha mostrado adecuación para su factorización $(\mathrm{KMO}=$ 0,$\left.73 ; \chi^{2}=231,30 ; p \leq 0,001\right)$ y se han obtenido tres factores que explican en conjunto el $58,6 \%$ de la varianza. El primer factor explica un $33,4 \%$ de la varianza en el que aparecen con pesos factoriales de relevancia (entre 0,44 y 0,81) cinco ítems: 1, 4, 7, 8 y 9. Dada su agrupación ha sido definido como "Disponibilidad y confianza en el equipo". Si atendemos a la clasificación de los ítems que propusieron los autores de la escala, tres hacen alusión a la dimensión satisfacción con el equipo -DSE- (1, 4 y 8) y dos a la dimensión satisfacción con el programa -DSP- (7 y 9).

En el segundo factor, denominado "Falta de confianza e insatisfacción con el equipo y el programa", también saturan con pesos moderados-altos (entre 0,42 y 0,79 ) cinco ítems $(1,3,5,6$ y 10$)$, que al igual que en el factor anterior, tres hacen referencia a la DSE y dos a la DSP. En total este factor explica el $13,9 \%$ de la varianza.

El tercer factor, con un $11,2 \%$ de la varianza, queda saturado de forma más relevante por cuatro ítems $(2$, 4,5 y 10), con cargas factoriales entre 0,41 y 0,78 . Este factor ha sido denominado "Recepción de información y motivación para el tratamiento".

Tabla 2. - Análisis Fiabilidad (Consistencia Interna) en función de los Factores Originales o los hallados en el estudio

Extracción

Factores (Denominación)

Items

$\alpha$

CCI

Factorial

\section{Original}

Equipo terapéutico

Programa terapéutico
$1,3,4,5,8$

0,65

0,85

$2,6,7,9,10$

0,59

0,81

\section{Actual}

Disponibilidad del equipo y confianza hacia el equipo

$1,4,7,8,9$

0,62

0,80

Falta de confianza e insatisfacción con equipo y programa

Recepción de información y motivación para el tratamiento

$1,3,6,10$

0,71

0,85

$2,5,10$

0,56

0,81

Total ITEMS TPQ-10

1 a 10

0,77

0,83

$\alpha$ : Alpha de Cronbach (Índice de Consistencia interna); CCl: Coeficiente de Correlación Intraclase (Índice de Concordancia) 
El análisis de consistencia interna (alpha de cronbach) y concordancia (Coeficiente de Correlación Intraclase - CCI) tanto de las escalas totales como por dimensiones, sean las teóricas originales o las encontradas en el análisis factorial realizado, aparecen en la tabla 2.

El índice de fiabilidad alcanzado para la escala total ha sido de un valor alpha de cronbach de 0,77 , que puede ser considerado como aceptable ${ }^{(10)}$. Atendiendo a las dimensiones teóricas, los ítems que componen la satisfacción con el equipo alcanzan un coeficiente de consistencia interna de 0,65, siendo de 0,59 para el caso de la dimensión de satisfacción con el programa; ambos índices han de considerarse como moderados. $\mathrm{Si}$ atendemos a las tres dimensiones encontradas tras la factorización del TPQ-10 en nuestro estudio, se observan coeficientes alpha de entre 0,56 y 0,71; dos de ellos considerados como moderados y uno que alcanza un valor moderado-alto que resulta aceptable.

Por otra parte, el valor observado del $\mathrm{CCl}$ para la escala total ha sido de 0,83 ; lo que implica que el porcentaje de varianza atribuible al desacuerdo entre las observaciones realizadas por los sujetos en dos momentos diferentes es del $17 \%$. Estos valores han de interpretarse como apoyo a una fiabilidad testretest alta.

\section{DISCUSIÓN}

Los resultados obtenidos confirman convenientemente la fiabilidad del TPQ en su consistencia interna y reproductibilidad, pero no apoyan la estructura factorial propuesta por sus autores ${ }^{(9)}$. La obtención de tres factores contradice la propuesta bidimensional de la escala original, más cuando la agrupación de ítems en cada uno de los factores resulta mixta -es decir, ninguno de los factores encontrados es coincidente con alguna de las dimensiones originales propuestas-. La estructura bifactorial original resulta sugerente por su diferenciación entre la valoración de los recursos humanos vs los recursos de estructura de un programa, siendo aceptable la validez aparente de tal diferenciación, pero nuestros datos apuntan que el fenómeno subyacente a la percepción del tratamiento por parte de los usuarios es más complejo y multicomponente. La diferencia de resultados encontrados por los autores del TPO y los ofrecidos en este estudio, bien pudieran ser atribuidos a cuestiones de tipo metodológico tales como la selección de la muestra (más de seis meses vs 15 días en tratamiento), el contexto de tratamiento (programas residenciales vs tratamiento ambulatorio), o a la variabilidad interestudios / intermuestras que sufre la aplicación de las técnicas exploratorias de componentes principales. Si bien los resultados obtenidos no permiten invalidar el modelo teórico bidimensional del TPQ, si sugieren la posibilidad de revisar los constructos subyacentes al fenómeno de la percepción y satisfacción de los usuarios respecto al tratamiento que reciben e incorporarlos a la versión delTPQ. A este respecto, se hacen necesarios más estudios, con utilización de muestras variadas que permitan agrupar el conjunto de usuarios de drogas en tratamiento, en aras a buscar la reproductibilidad de resultados, lo que permitiría una mayor validez en la confirmación de la validez de constructo delTPQ.

Por otra parte, si bien el TPQ no reproduce en nuestra muestra la estructura teórica original, sí obtiene índices de bondad psicométrica. La consistencia interna (fiabilidad) es mayor cuando se utiliza la escala total que cuando se recurre a sus dimensiones, sean éstas las propuestas originariamente o las halladas con nuestros datos; en el primer caso el coeficiente de cronbach ha de ser considerado alto (alpha $=0,77$ ), mientras que en el caso de las dimensiones tiende a ser moderado (entre 0,51 y 0,71). Donde sí muestra el TPQ su mayor adecuación es en la consistencia de su reproductibilidad ya que en todos los casos los $\mathrm{CCl}$ están por encima de 0,80. Estos resultados apoyan la utilización del TPQ como un instrumento estable.

La principal limitación del estudio radica en el tamaño muestral utilizado ya que muestras pequeñas tienden a carecer de precisión en el ajuste de los índices estadísticos. Otra limitación metodológica se encuentra en la extensión del propio instrumento. Algunos autores ${ }^{(10)}$ plantean la necesidad de considerar, en la selección de los factores, valores propios superiores a 1,5 cuando se trabaja con muestras relativamente pequeñas respecto al número de ítems de la escala. En este caso, en nuestro estudio del TPQ tan solo debiéramos conservar el primer factor, lo cual acotaría de forma importante la varianza del fenómeno a explicar y, por otra parte, excluiría cinco ítems de dicha explicación. Otra forma de comprobar la idoneidad de la estructura bidimensional propuesta por el grupo de Marsden es aplicar técnicas de análisis factorial confirmatorio, pero para ello también sería necesario ampliar el número de efectivos. Este es el objetivo que se ha planteado nuestro grupo para próximos estudios delTPQ.

Como conclusión, consideramos que el TPQ constituye un instrumento muy cómodo y manejable para la valoración del tratamiento recibido por los usuarios de programas de drogas y muestra una adecuada idoneidad psicométrica respecto a su fiabilidad y consistencia test-retest. Sin desestimar la propuesta bidimensional original, altamente sugerente tanto a nivel teórico como aplicado, los resultados nos invitan a pensar que la valoración de la satisfacción con el tratamiento es multidimensional y que se precisa avanzar en esta línea de trabajo a través de la ejecución de 
nuevos estudios y la aplicación de técnicas de análisis más sofisticadas.

\section{REFERENCIAS}

1. Caminal J. La medida de la satisfacción: un instrumento de participación de la población en la mejora de la calidad de los servicios sanitarios. Rev Calidad Asistencial 2001; 16: 276-279.

2. Fitzpatrick R. Surveys of patient satisfaction: II-Designing a questionnaire and conducting a survey. BMJ 1991; 302: 1129-1132.

3. Mira JJ, Aranaz J. La satisfacción del paciente como una medida del resultado de la atención sanitaria. Med Clin (Barc) 2000; 114 (Supl 3): 26-33.

4. Pascoe CR. Patient satisfaction in primary healt care: A literature review and analysis. Eval Program Plann 1983; 6: 185-210.

5. James RE, Lindsay WL. Administración y control de calidad. México: Grupo Editorial Iberoamericano; 1995.

6. Marsden J, Nizzoli U, Corbelli C, Margaron H, Torres MA, Castro del Prado I, Stewart D, Gossop M. Fiabilidad del "Maudsley addiction profile" (MAP, ERIT- Versión) del "Treatment perceptions questionnaire" (TPQ) en Italia, España y Portugal para la evaluación de tratamientos. Adicciones 2001; 13: 217-227.

7. Rodríguez M, Jiménez-Lerma JM, Iraurgi I, Murua F, Bacigalupe L, Chavarri MR, Balsategi B. Evaluación de la Satisfacción con el Tratamiento en un Centro Ambulatorio de Drogodependencias a través del "Treatment Perception Questionnaire" (TPQ). Adicciones 2002; 14 (4): 1-8.

8. Pérez de los Cobos J, Valero S, Haro G, Fidel G, Escuder G, Trujols J, Valderrama JC. Development and psychometric properties of the Verona Service Satisfaction Scale for methadone-treated opioid-dependent patients (VSSS-MT). Drug Alcohol Depend 2002; 68(2): 209-214.

9. Marsden J, Stewart D, Gossop M, Rolfe A, Bacchus L, Griffiths P, Clarke K, Strang J. Assessing client satisfaction with treatment for substance use problems: Development of the Treatment Perceptions Questionnaire (TPQ). Addiction Research 2000; 8 (5): 455-470.

10. Nunnally JC, Berstein IJ. Teoría psicométrica. México:

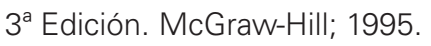

11. Bartko, J.J. The intraclass correlation coefficient as a measure of reliability. Psychological Repport 1966; 19: 3-11.

12. Prieto, L., Lamarca, R., Casado, A., Alonso, J. The evaluation of agreement on continuous variables bay the intraclass correlation. Journal Epidemiology and Community Health, 1997; 51(5): 579-580.

13. Prieto, L., Lamarca, R., Casado, A. La evaluación de la fiabilidad en las observaciones clínicas: el coeficiente de correlación intraclase. Med Clín (Barc) 1998; 110: 142-145.

14. Norusis MJ. Statistical Package for the Social Sciences. Release 9. Chicago: SPSS Inc.; 2000. 